\title{
A Global Search Reveals Epistatic Interaction Between QTL for Early Growth in the Chicken
}

\author{
Örjan Carlborg, ${ }^{1,4}$ Susanne Kerje, ${ }^{1}$ Karin Schütz, ${ }^{2}$ Lina Jacobsson, ${ }^{1}$ Per Jensen, ${ }^{2}$ \\ and Leif Andersson ${ }^{1,3}$ \\ ${ }^{1}$ Department of Animal Breeding and Genetics, Swedish University of Agricultural Sciences, S-751 24 Uppsala, Sweden; \\ ${ }^{2}$ Department of Animal Environment and Health, Section of Ethology, Swedish University of Agricultural Sciences, \\ S-532 23 Skara, Sweden
}

\begin{abstract}
We have identified quantitative trait loci (QTL) explaining a large proportion of the variation in body weights at different ages and growth between chronological ages in an $F_{2}$ intercross between red junglefowl and White Leghorn chickens. QTL were mapped using forward selection for loci with significant marginal genetic effects and with a simultaneous search for epistatic QTL pairs. We found 22 significant loci contributing to these traits, nine of these were only found by the simultaneous two-dimensional search, which demonstrates the power of this approach for detecting loci affecting complex traits. We have also estimated the relative contribution of additive, dominance, and epistasis effects to growth and the contribution of epistasis was more pronounced prior to 46 days of age, whereas additive genetic effects explained the major portion of the genetic variance later in life. Several of the detected loci affected either early or late growth but not both. Very few loci affected the entire growth process, which points out that early and late growth, at least to some extent, have different genetic regulation.
\end{abstract}

[Supplemental material is available online at www.genome.org.]

During the past decade, numerous studies have been performed to increase the understanding of the molecular genetic mechanisms behind complex traits. Various traits have been studied, ranging from disease phenotypes in humans to production traits in farm animals. Many major genes and quantitative trait loci (QTL) have been identified and the molecular mechanism behind several of these has been identified (Andersson 2001; Flint and Mott 2001; Korstanje and Paigen 2002). Domestic animals harbor a large resource of functional mutations affecting a wide range of phenotypic traits such as growth, reproduction, behavior, and resistance to disease. During thousands of years of domestication and numerous generations of intense artificial selection, the frequency of QTL alleles with desired effects on these traits have increased in the domesticated lines. Intercrosses between the wild ancestor and a domesticated line can be used to detect genomic regions harboring genes, which have been under selection during domestication. The red junglefowl is the wild ancestor of the domesticated chickens we use for egg and meat production today. The junglefowl and the domesticated chickens differ dramatically for many traits (e.g., growth). We have generated a large intercross between the red junglefowl and a White Leghorn laying hen comprising more than $800 \mathrm{~F}_{2}$ animals and used this for mapping QTL affecting behavior (Schütz et al. 2002) and production traits (S. Kerje, Ö. Carlborg, K. Schütz, L. Jacobsson, C. Hartmann, P. Jensen, and L. Andersson, in prep.). In the latter study, we identified 13 QTL

\section{${ }^{3}$ Corresponding author.}

E-MAIL Leif.Andersson@bmc.uu.se; FAX +46-18 4714833. ${ }^{4}$ Present address: Roslin Institute, Roslin, Midlothian, EH25 9PS, Scotland.

Article and publication are at http://www.genome.org/cgi/doi/10.1101/ gr.528003. affecting growth using a standard one-dimensional QTL analysis. Some of the detected QTL had very large effects and these loci explained a large proportion of the variation in adult body weight in this cross suggesting an excellent power for QTL detection. The present study involves a more thorough dissection of the genetic components, including a search for epistatic interactions, for variation in body weight at different ages and growth between chronological ages for the White Leghorn and the red junglefowl.

Lilja (1983) compared growth rates in birds with varying growth rate capacities. He showed that a high growth rate capacity is characterized by a rapid early development of the digestive organs and the liver, whereas a low growth rate is characterized by a rapid early development of the pectorals and feathers. Other studies (Lilja et al. 1985; Katanbaf et al. 1988; Lilja and Marks 1991; Nitzan et al. 1991; Nir et al. 1993) have shown that selection for high growth rate in chickens and quail is linked to an increase in the relative size of the digestive organs. These studies show that difference in growth pattern is under genetic control, and that variation exists within species. In general, growth can be caused by cell division, increase in cell size, or deposition of extra-cellular material. Muscular growth after hatching is the result of an increase in size of the muscle cells, as basically all cell division takes place during embryonal development. For the internal organs, for example, liver or kidney, both the numbers of cells and the sizes of cells can change throughout the life cycle. The number of feathers is decided during the embryonal stage, but new feathers can grow throughout life. Deposition of fat in domestic animals normally takes place during late growth, where it becomes the major contributor to increases in body mass (Björnhag et al. 1994).

The most frequently used statistical methods for genetic analysis of experimental crosses only model the marginal ge- 
netic effects (additive/dominance) of individual loci, thus ignoring interactions between QTL (epistasis). Epistasis has been considered in several studies, and then either by testing for epistasis between QTL detected by their marginal effects (e.g., Chase et al. 1997) or by using one-dimensional searches with an epistatic model, while including markers to control background genetic effects (e.g., Fijneman et al. 1996). Epistasis has also been evaluated experimentally and found to be an important contributor to quantitative traits (Mackay 2001). More recently, several methods for mapping epistatic QTL have been proposed. All of the proposed methods use a genetic model including interaction terms for pairs of QTL, in order to increase the power to detect interacting QTL and to better understand the importance of epistasis for complex traits. The methods are either based on one-dimensional genome scans (Jannink and Jansen 2001), simultaneous searches on preselected genome regions (Kao et al. 1999) or simultaneous mapping of epistatic QTL in a grid based on markers (Shimomura et al. 2001). We have recently proposed a method for simultaneous mapping of genome-wide epistatic QTL based on a genetic algorithm (Carlborg et al. 2000; Carlborg and Andersson 2002). We used a slightly modified version of that method in this study to map epistatic QTL for growth in the experimental red junglefowl/White Leghorn cross. The aim was to unravel the molecular basis for growth differences between these divergent lines, by estimating the number of QTL, their action, and interactions. We also intended to compare the performance of our proposed simultaneous mapping method for detecting epistatic QTL to a standard QTL mapping method, and to evaluate the increase in power when analyzing experimental data.

\section{RESULTS}

\section{Information Content and Genomic Coverage by the Current Genetic Map}

The results reported here are based on an initial genome scan using 105 markers across the chicken genome with an average marker density of $24.4 \mathrm{cM}$. The information content varies among the markers, where some markers are fully informative and others have an information content $<0.5$. Because of low information content and the relatively sparse genetic map, there are regions in the genome where the power to detect QTL of moderate effects is rather low. We have therefore chosen to also use the QTL detected at a slightly lower $20 \%$ genome-wide significance for the estimation of the genetic variance explained by QTL detected in this study to avoid a high rate of type II errors. Because we have carried out genome scans for nine growth-related traits, we expect to obtain about two false positives. We identified altogether 32 QTL significant at the 20\% level (see below). Thus, we conclude that a majority of these QTL represents true effects.

\section{A Simultaneous Search for Epistatic QTL Pairs Increases the Power to Detect QTL}

The number of detected genomic regions with genome-wide significant effects on at least one of the growth-related traits in this study was almost doubled by including a simultaneous mapping step and a randomization test to detect epistatic QTL pairs (from here referred to as SIM). The increase in power (here measured as the number of detected loci) was $+70 \%$ when using a $5 \%$ genomewide significance threshold and $+145 \%$ when using a $20 \%$ significance threshold.

We mapped QTL for the nine growth-related traits in this study. QTL detected for different traits were considered as the same QTL if their location estimates were in the same marker bracket. A total of 32 QTL were found in the genome when using a 20\% significance threshold as suggestive evidence for QTL (Table 1). Twelve QTL were identified using both forward selection for QTL and by the SIM method, and 19 regions were only identified using SIM. Using the more stringent 5\% genome-wide significance threshold, 22 regions were detected in total, 12 of these by both forward selection and SIM, while one region was only detected by forward selection and nine regions were only detected by SIM. In Table 1 , we also report the markers flanking the interval in which each quantitative trait locus is located (or the marker at which the QTL are located) together with the information content at that location.

The growth traits analyzed in this study are correlated. The correlation is intermediate for adjacent body weights and growth measures (0.5-0.6) and relatively low otherwise $(<0.25)$, which is an indication that the measured growth traits to some extent are distinct traits. We found that a majority of the QTL only affected one or two of the traits. Several of the QTL, which only affected one trait, were only detected at a $20 \%$ significance threshold (Fig. 1), which indicates that the QTL have smaller effects and only can be detected during the growth phase where they have the largest impact. Most of the QTL affecting three or more traits remain when the significance threshold was increased, which indicates that they have a larger and more general effect in the growth process.

Figure 2 shows the complete results from the mapping procedure for growth between 8 and 46 days of age (early growth), including the location of the individual QTL detected by forward selection interval mapping and pairs identified by simultaneous mapping of epistatic QTL pairs. The figure also shows whether the QTL was detected using forward selection, SIM, or by both methods. The complete data for all other traits in the study are available as a supplement to this article.

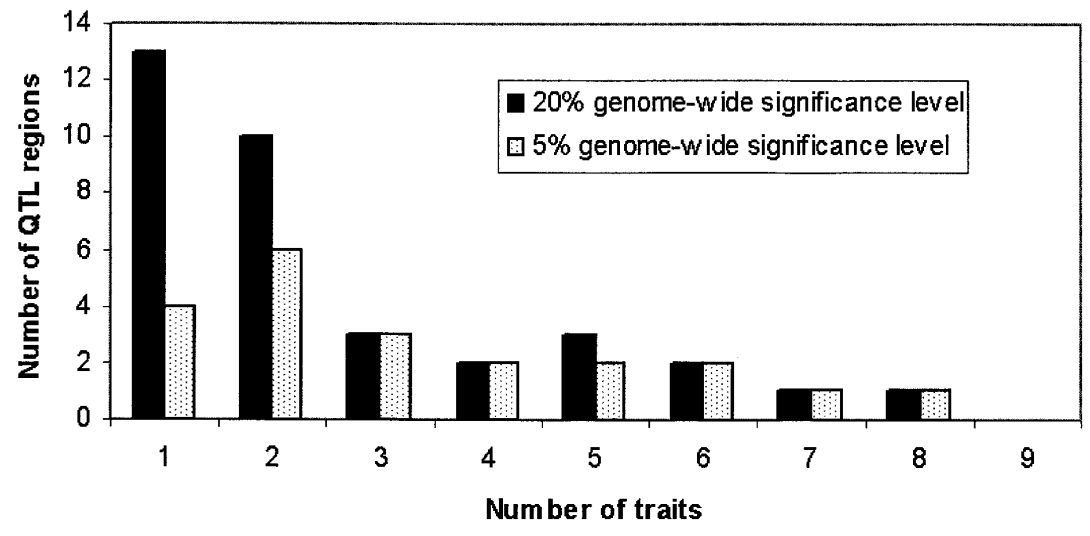

Figure 1 The number of quantitative trait loci affecting one to nine of the traits in the study at a $5 \%$ and $20 \%$ genome-wide threshold as determined by randomization testing.

\section{Genome Research}




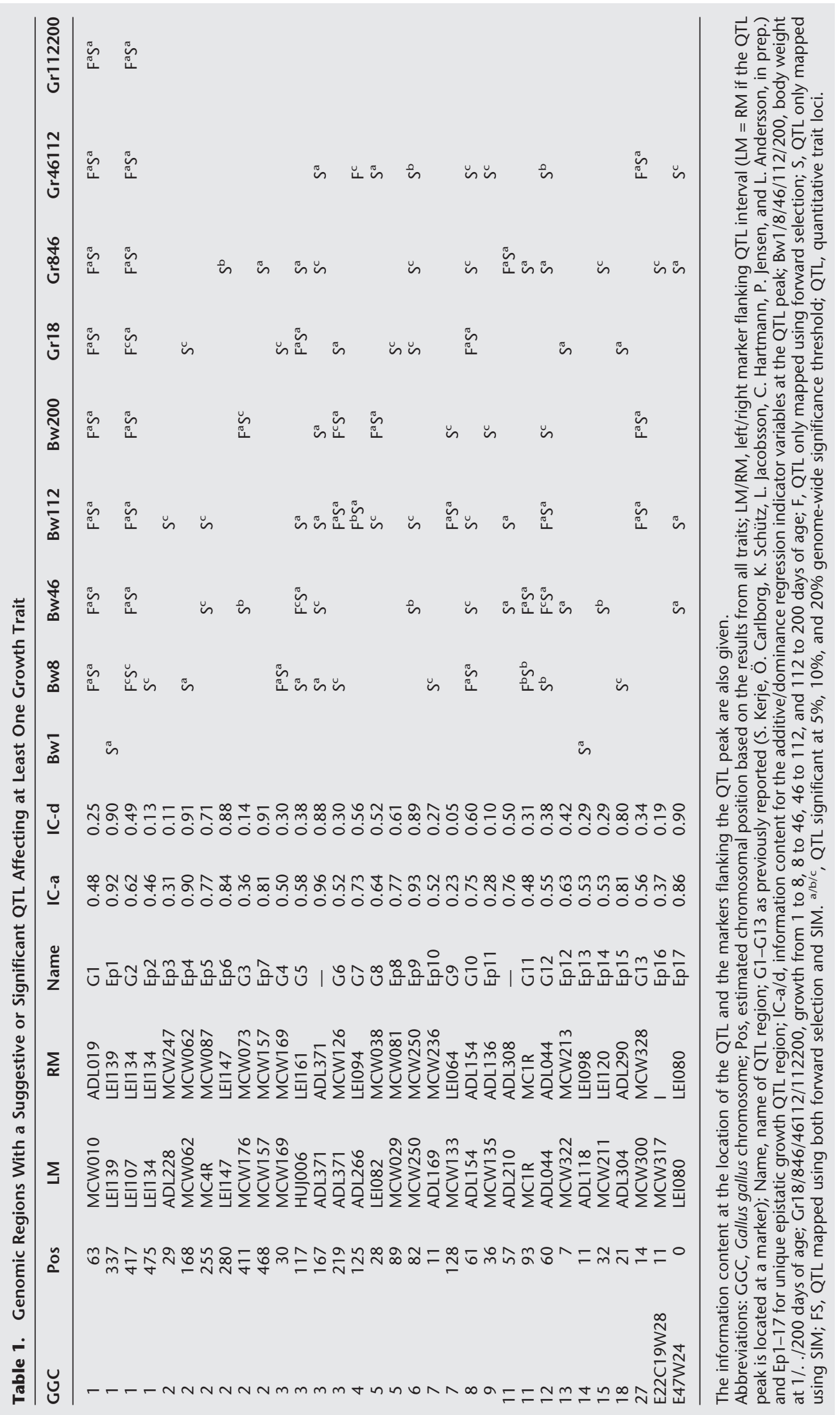




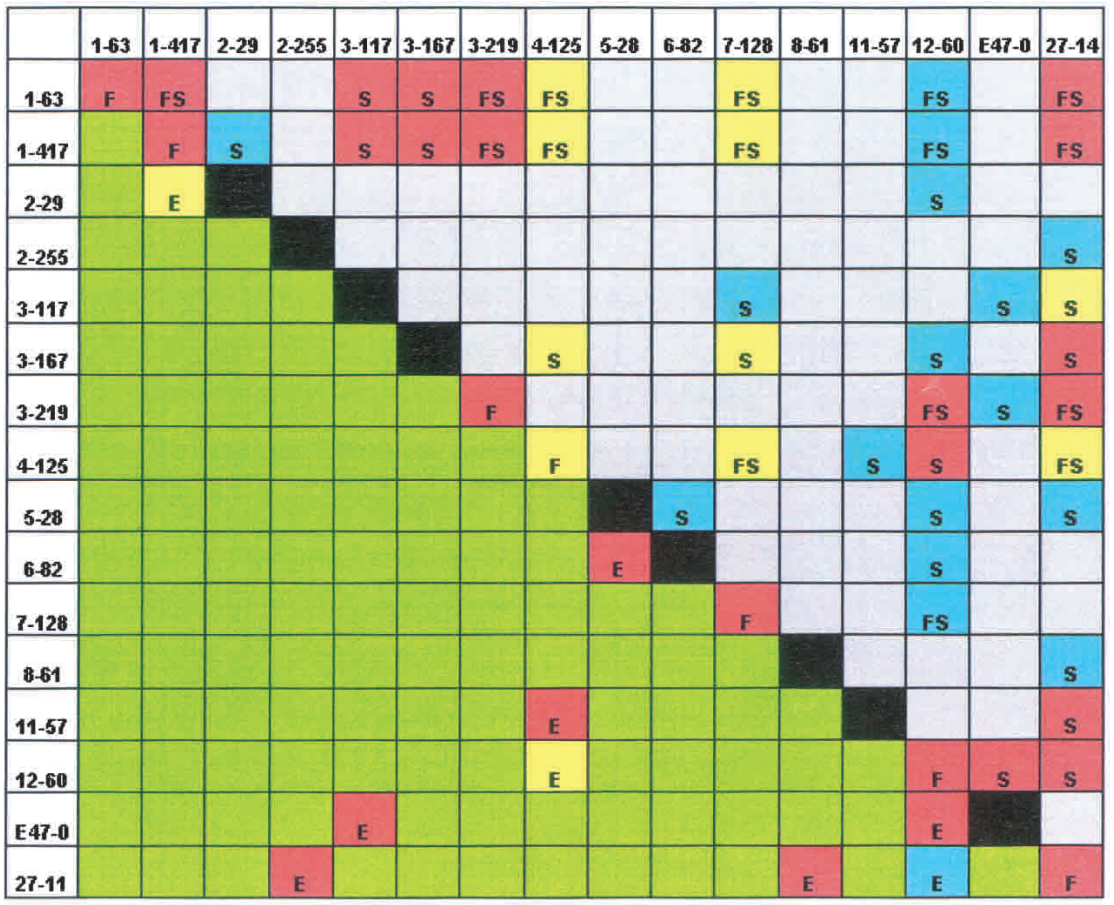

\section{Significant using $5 \%$ genome-wide threshold Significant using $10 \%$ genome-wide threshold Significant using $20 \%$ genome-wide threshold}

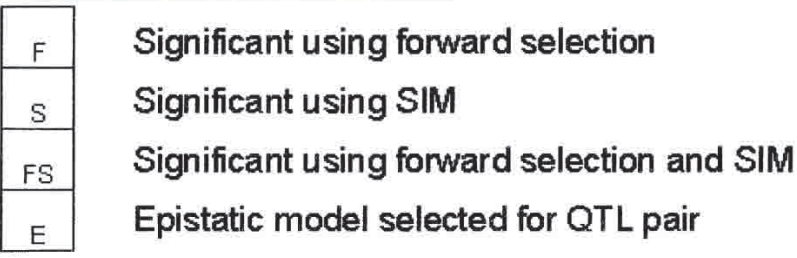

Figure 2 All quantitative trait loci (QTL) for body weight at 112 days of age detected by forward selection and SIM. The QTL detected using a forward selection search are presented on the diagonal (F). All QTL pairs that were detected by SIM (S) or both forward selection and SIM (FS) are presented above the diagonal. QTL pairs where the epistatic model was selected by a randomization test are presented below the diagonal (E). The results are given for a 5\%, 10\%, and 20\% genome-wide significance threshold. The full designation of linkage group E47 is E47W24.

\section{Estimated Phenotypic Effects of Epistatic QTL Pairs}

The obtained indicator regression variables for interacting QTL pairs were used to estimate least square means for all nine possible genotypes for pairs of interacting QTL (each locus was assumed to segregate for two alleles inherited from the red junglefowl and the White Leghorn founders, respectively). The epistatic QTL pair affecting weight at hatch (Bw1) is used to illustrate the results obtained in this type of analysis. Our previous one-dimensional search revealed no significant QTL for hatch weight. The results presented in Figure 3 and Table 2 show that the two opposite homozygotes (chr. 1:337) $J / J-($ chr. 14:11) $L / L$ and (chr. 1:337) $L / L-($ chr. 14:11) $J / J$ have a reduction in hatch weight of about 10\% (2.2-4.7 gr) compared with the population-matched homozygotes (J/I-J/J and $L / L-L / L)$, while the remaining five genotypes tended to show intermediate phenotypes. The results suggest some form of physiological incompatibility in the opposite homozygotes. This could, for instance, reflect mutations in a receptor and a ligand, inhibiting an appropriate interaction. This interacting QTL pair is of interest in relation to the fact that a reduced fitness may be observed in the $\mathrm{F}_{2}$ generation of wide crosses, a phenomenon that has been attributed to possible epistatic interaction (Falconer 1981). It is worth noticing that the $\mathrm{F}_{2}$ generation of our intercross in fact had an unexplained reduced early growth (from day 1 to day 46) compared with both parental populations (S. Kerje, Ö. Carlborg, K. Schütz, L. Jacobsson, C. Hartmann, P. Jensen, and L. Andersson, in prep.) that may reflect this type of negative epistatic interaction.

\section{Evidence for Significant Interactions Between QTL Pairs Affecting Growth}

Table 3 shows how often an additive/dominance and an epistatic QTL model were selected for all QTL pairs significant at the $5 \%$ and $20 \%$ significance thresholds. When a 5\% threshold was used for selecting an epistatic model, 12 QTL pairs were significant. Suggestive evidence at a $20 \%$ significance level exists for an additional 51 pairs. The fact that the number of QTL pairs exceeds the total number of QTL $(n=32)$ is because some QTL show multiple interactions. An epistatic model was selected for at least one QTL pair for all traits except body weight at 200 days and growth from 112 to 200 days. The table also indicates how often the simultaneously detected QTL pair included two, one, or no QTL, which were significant by forward selection QTL mapping. In total, 12 (28) QTL pairs were detected using a 5(20)\% genome-wide significance threshold, where none of the QTL had significant marginal effects. 


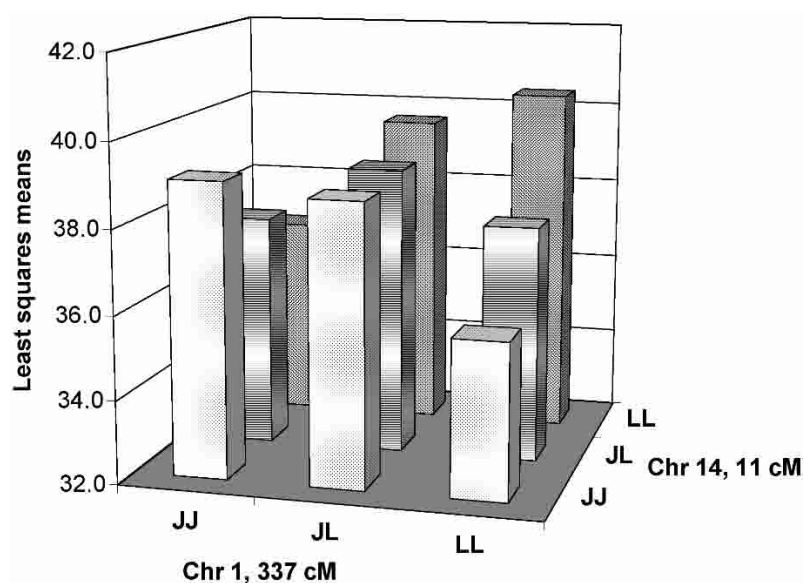

Figure 3 Least square means for the nine possible genotypes for the interacting quantitative trait loci pair affecting weight at hatch. Each locus was assumed to segregate for two alleles inherited from the red junglefowl $(J)$ and the White Leghorn $(L)$ founders, respectively.

\section{Simultaneously Mapped QTL Increase the Variance Explained by the QTL Model}

We compared the residual variances explained by those QTL that were significant in the forward selection QTL mapping procedure and by SIM at a 5\% genome-wide significance level. The comparison of the residual variance explained gives another measure of the potential increase in power by using the simultaneous QTL mapping procedure (Fig. 4). The residual variance explained ranged from $1.6 \%$ to $32.4 \%$ for the QTL mapped by forward selection and from $4.8 \%$ to $35.0 \%$ for the simultaneously mapped QTL. Simultaneous mapping, on average, explains an additional $4.9 \%$ of the residual variance, with a range from $0 \%$ to $10.4 \%$. Larger increases were obtained for early growth, whereas no improvement was obtained for late growth.

\section{Partitioning of the Phenotypic Variance}

We estimated the proportion of the phenotypic variance in the $\mathrm{F}_{2}$ generation, which could be explained by systematic environmental factors (sex and batch) and genetic components (additive, dominance, and epistatic effects) for significant and suggestive QTL. The relative contribution of the genetic factors to the total variance of the traits is given for all traits in Figure 5. The genetic variance explained from $4 \%$ to $26 \%$ of the total phenotypic variance in different traits. There were higher relative contributions from genetic factors and batch effects to early growth, whereas the sex of the individual was the major contributor to the phenotypic variance at the later stages of growth, in particular during the period from 46 to 112 days of age.

\section{Partitioning of the Genetic Variance Explained by QTL}

Figure 5 shows the partitioning of the genetic variance into additive, dominance, additive by additive, additive by dominance, and dominance by dominance terms. The genetic contribution to early growth is rather low. On the other hand, a large part of the genetic variance that can be explained is from epistasis. For the QTL detected, 80\% of the genetic variance for hatch weight and 70\% for growth from 1 to 8 days of age are caused by epistasis. The relative contribution of epistasis decreases for later growth, $15 \%-40 \%$ of the total genetic variance, and for late growth almost all of the genetic variance is additive. The contribution of dominance to the total genetic variance is constant, around 5\%-10\%, for all traits except late growth.

\section{The Distribution of the Additive and Dominance Effects for the Mapped QTL}

To further explain the genetics behind the growth traits, we estimated the additive and dominance effects for the 15 QTL for body weight at 112 days, which were significant at a $20 \%$ genome-wide significance threshold (Fig. 6). The size of the additive effects show that there is a limited number of QTL that have a large additive effect on the trait, and that several other QTL have smaller effects on the trait. Dominance seems to be important for many loci and especially those with smaller additive effects. Although the figure suggests overdominance at several QTL, there is only significant evidence for overdominance at one locus (number 14).

\section{DISCUSSION}

In this report, we have shown that simultaneous mapping of QTL pairs allowing epistatic interaction increases the power to detect QTL in experimental line crosses. Both the number of detected QTL regions and the residual variation explained by the QTL increased. The number of QTL significant at the $5 \%$ genome-wide level for at least one growth trait increased from 13 using a standard one-dimensional QTL search to 22 using our simultaneous search for QTL pairs (SIM). The additional QTL detected by simultaneous mapping caused a substantial increase in the residual variance explained for early growth, but only marginal improvement for late growth (Figs. $4,5)$. Therefore, we conclude that the value of using a simultaneous search will depend on how important epistatic interaction is for the trait under study. Furthermore, a large sample size is required to obtain reasonable power to detect epistasis. To obtain high power, we recommend a sample of at least 500 $\mathrm{F}_{2}$ individuals.

The use of an epistatic model in QTL mapping means that additional degrees of freedom are introduced in the mapping procedure. This has potential benefits, as well as drawbacks. The increased freedom increases the power to detect interacting QTL that are difficult to detect using ordinary QTL mapping methods. There is, however, a risk that the method is more sensitive to inconsistencies and skewness (such as segregation distortion) in the genetic and phenotypic data, as additional degrees of freedom are introduced in the genetic

\begin{tabular}{|c|c|c|c|}
\hline \multirow{2}{*}{$\begin{array}{l}\text { QTL genotype } \\
\text { Chr. 1, } 337 \text { cM }\end{array}$} & \multicolumn{3}{|c|}{ QTL genotype Chr. 14, 11 cM } \\
\hline & $J / J$ & $J / L$ & $L / L$ \\
\hline$J / J$ & $39.0 \pm 0.7$ & $38.7 \pm 0.6$ & $35.7 \pm 0.9$ \\
\hline$J / L$ & $37.6 \pm 0.5$ & $38.9 \pm 0.4$ & $37.7 \pm 0.6$ \\
\hline$L / L$ & $36.8 \pm 0.7$ & $39.6 \pm 0.6$ & $40.4 \pm 0.8$ \\
\hline
\end{tabular}

J, allele inherited from the red junglefowl; L, allele inherited from White Leghorn; QTL, quantitative trait loci. 
Table 3. Number of QTL Pairs Identified by a Simultaneous Mapping Strategy for Epistatic QTL Pairs

\begin{tabular}{|c|c|c|c|c|c|c|c|c|c|c|c|c|}
\hline & \multicolumn{6}{|c|}{$5 \%$ Genome-wide significance } & \multicolumn{6}{|c|}{$20 \%$ Genome-wide significance } \\
\hline & \multirow{2}{*}{$\begin{array}{l}\text { no. of pairs } \\
\text { by SIM }\end{array}$} & \multicolumn{2}{|c|}{ selected model } & \multicolumn{3}{|c|}{ detected by $\mathrm{F}$} & \multirow{2}{*}{$\begin{array}{l}\text { no. of pairs } \\
\text { by SIM }\end{array}$} & \multicolumn{2}{|c|}{ selected model } & \multicolumn{3}{|c|}{ detected by $F$} \\
\hline & & $A+D$ & E & 2 & 1 & 0 & & $A+D$ & E & 2 & 1 & 0 \\
\hline Bw1 & 1 & 0 & 1 & 0 & 0 & 1 & 1 & 0 & 1 & 0 & 0 & 1 \\
\hline Bw8 & 6 & 5 & 1 & 2 & 3 & 1 & 20 & 9 & 11 & 9 & 7 & 4 \\
\hline Bw46 & 11 & 9 & 2 & 3 & 3 & 5 & 30 & 21 & 9 & 10 & 14 & 6 \\
\hline Bw112 & 16 & 11 & 1 & 7 & 9 & 0 & 41 & 32 & 9 & 17 & 22 & 2 \\
\hline Bw200 & 10 & 10 & 0 & 8 & 2 & 0 & 22 & 18 & 4 & 14 & 8 & 0 \\
\hline Gr18 & 7 & 3 & 4 & 2 & 4 & 1 & 14 & 2 & 12 & 4 & 8 & 2 \\
\hline Gr846 & 7 & 4 & 3 & 3 & 1 & 3 & 19 & 9 & 10 & 3 & 7 & 9 \\
\hline Gr46112 & 5 & 5 & 0 & 3 & 1 & 1 & 14 & 7 & 7 & 3 & 7 & 4 \\
\hline Gr112200 & 1 & 1 & 0 & 1 & 0 & 0 & 1 & 1 & 0 & 1 & 0 & 0 \\
\hline
\end{tabular}

The table shows for how many of the detected pairs that a marginal effects model including additive and dominance effects $(A+D)$ and an epistatic QTL model (E) were selected, based on a model selection randomization test. The table also gives the number of times two, one or none of the QTL in the detected pair also was detected with forward selection (F) using a marginal effects model.

QTL, quantitative trait loci.

model. We have tried to minimize the risk of spurious associations by using randomization testing to obtain a null distribution for significance testing. Randomization testing should give an elevated threshold if inconsistencies would affect the mapping results. We have also manually rechecked the marker and phenotype data for each implied QTL. This includes following the segregation of the markers closest to the estimated QTL position, testing for segregation distortion at the proposed QTL locations as described by Knott et al. (1998), and plotting the distribution of the $a, d, a a, a d, d a$, and $d d$ regression indicator variables for each QTL. Based on this, we decided to remove one QTL region where substantial skewness was detected in the data. For the remaining regions, the mean and standard deviation for the estimates of information content and segregation distortion were similar for the QTL regions detected by their marginal effects and the epistatic QTL.

The relative contribution of epistasis to the total genetic variance in growth found in this study is similar to that found by Brockmann et al. (2000) in mice. We were, though, not able to explain as much of the total residual variation with the detected QTL. We work with an outbred line cross, instead of a cross between an inbred line and an extreme selection line, and can not raise the chickens under the same standardized conditions as laboratory mice. Therefore, we expect to have more unexplained residual variation present in our study. Another explanation might be that the selection for growth has not been as extreme in the White Leghorn as in the DU6i strain used by Brockmann et al. (2000), and therefore genetic heterogeneity within the lines used in our cross may reduce the power in the QTL analysis.

The distribution of the additive effects for the detected QTL for the growth traits showed that a limited number of QTL have very large additive effects and several smaller QTL have only marginal additive effects (Fig. 6). The result is simi- lar to that found by Zeng et al. (2000) using a cross between two divergent Drosophila species, and was expected because selection will strive to increase the frequency of alleles with large beneficial additive effects in the selected line. Several of the detected loci affected either early or late growth, but not both. Very few loci affected the entire growth process, which points out that early and late growth, at least to some extent, have different genetic regulation. This is in line with several previous studies suggesting that early and late growth, at least to some extent, are regulated by different genetic mechanisms. Falconer et al. (1978) found that two general physiological mechanisms seem to affect the increase in body size in mice, and these mechanisms appear to act at different life stages (Atchley and Zhu 1997). Several quantitative genetic studies of growth traits in mice have also indicated that individual genes might have opposite pleiotropic effects on early and late growth (Cheverud et al. 1983; Leamy and Cheverud 1984; Riska et al. 1984), and more recent QTL mapping experiments report that early and late growth in mice were af-

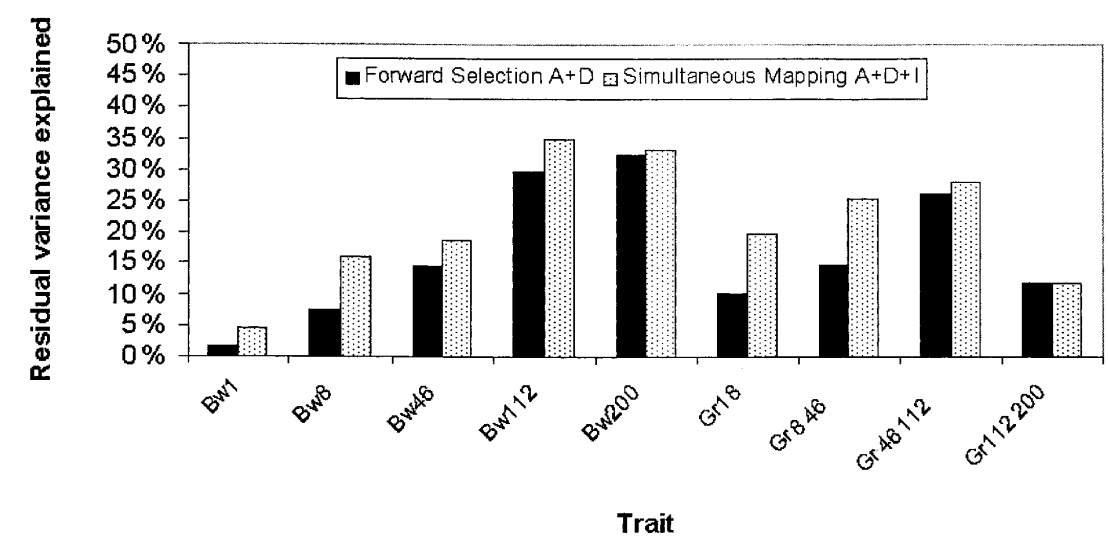

Figure 4 The residual variance explained by the marginal effects of the quantitative trait loci (QTL) mapped using forward selection and by the marginal and epistatic effects of the QTL mapped by SIM at a 5\% genome-wide significance level. A, additive effect; D, dominance effect; I, interaction; Bw1, body weight at day 1 ; Bw8, body weight at day 8; Bw46, body weight at day 46; Bw112, body weight at day 112; Bw200, body weight at day 200; Gr18, growth from day 1 to day 8; Gr846, growth from day 8 to day 46; Gr46112, growth from day 46 to day 112; Gr112200, growth from day 112 to day 200 . 

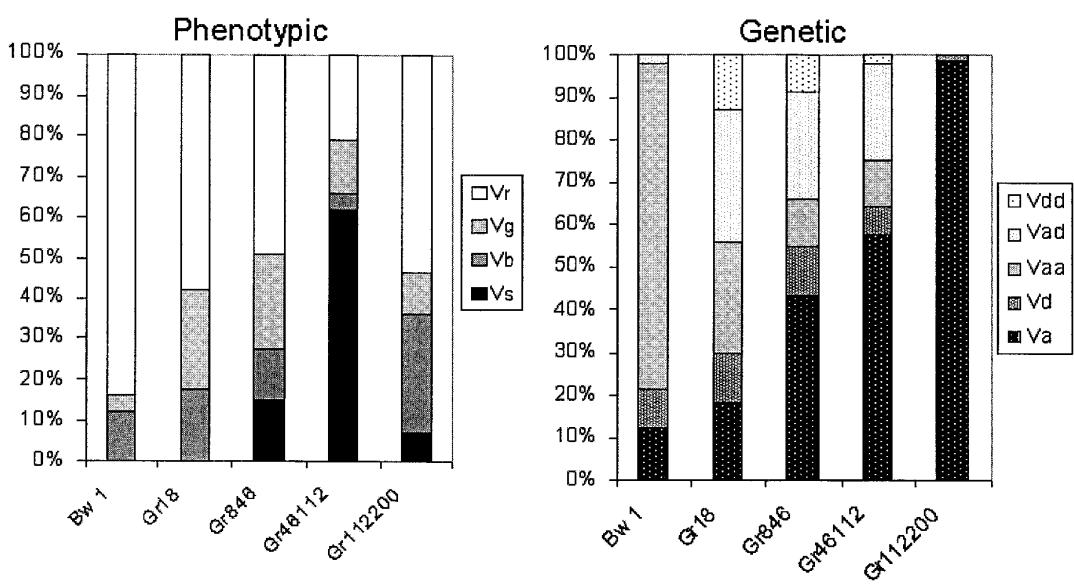

Figure 5 Partitioning of the total phenotypic $(P)$ and the genetic $(G)$ variance explained by the quantitative trait loci mapped by SIM at a $20 \%$ genome-wide significance level in the $F_{2}$ generation of a red junglefowl $\times$ White Leghorn intercross. $V r$, residual variance; $\mathrm{Vg}$, genetic variance; Vb, variance explained by batch effects; Vs, variance explained by sex effects; Vdd, dominance-by-dominance genetic interaction variance; Vad, additive-by-dominance and dominance-by-additive genetic interaction variance; Vaa, additive-by-additive genetic interaction variance; $\mathrm{Vd}$, dominance genetic variance; $\mathrm{Va}$, additive genetic variance. Abbreviations for traits are explained in the legend of Figure 4.

fected by distinct QTL, mapping to separate chromosome locations (Cheverud et al. 1996; Vaughn et al. 1999).

We were able to detect fewer loci that affected hatch weight and late growth (after 112 days), compared to the number of interacting and noninteracting genes affecting early and intermediate growth. Hatch weight was affected by two epistatic loci, explaining a relatively moderate proportion of the variance. This finding is in concordance with the findings by Hartmann (2002), who showed that the additive genetic effect on hatch weight is low. Most of the loci affecting late growth were involved throughout the entire growth process. H. Brändström, U. Gunnarsson, L. Andersson, C. Ohlsson, H. Mallmin, S. Larsson, and A. Kindmark, (in prep.) have mapped QTL for body composition in the same chicken population. Their study showed that those QTL that we have shown to affect the entire growth process caused an increased deposition of muscle tissue in the birds. These QTL could affect growth by either increasing the growth of muscle cells, or increasing the division of muscle cells during embryonal development, thus giving animals a greater potential for growth as a result of larger numbers of muscle cells. Growth prior to 46 days is affected by a large number of QTL. There is also a significant contribution of epistasis to the explained genetic variance during this period. This early growth is characterized by the development of internal organs and growth of feathers, and these growth processes are likely to be regulated by complex genetic networks. It is therefore not surprising that we in this study have found 22 significant and 10 additional suggestive QTL that affect the growth-related traits. The results indicate that the large difference in growth between the red junglefowl and the White Leghorn is under complex genetic control, suggesting that numerous physiological

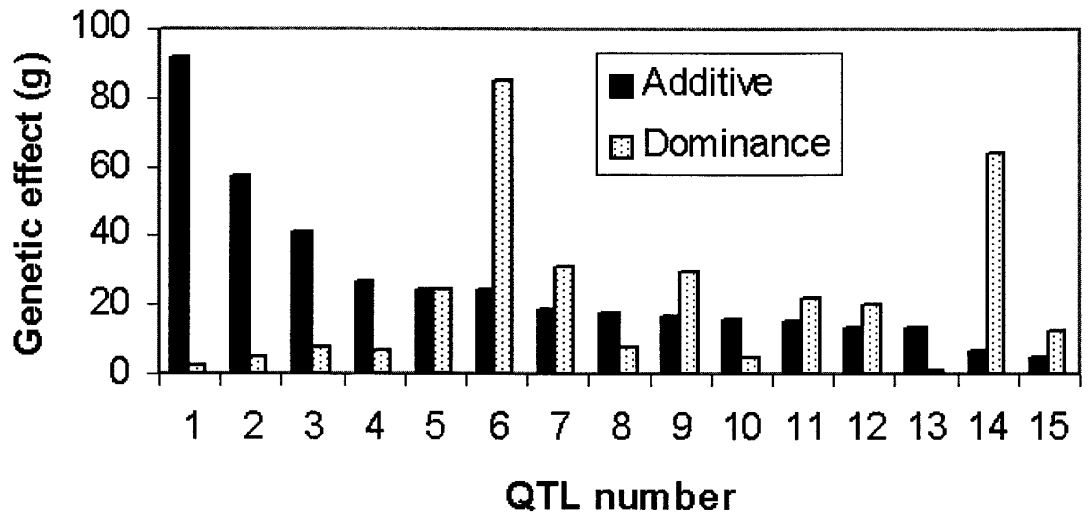

Figure 6 Additive and dominance genetic effects for the quantitative trait loci affecting body weight at 112 days that were mapped by SIM using a $20 \%$ genome-wide significance threshold, sorted by size of the additive effect. processes have been altered during selection. The intermediate growth (46-112 days) is where a main deposition of body mass takes place. Our analysis indicates that a relatively large number of genes are involved and that there is a relatively low contribution of epistasis. Even though a large number of genes are involved, the major effects on growth are caused by a rather limited number of genes, which additively affect deposition of muscle tissue (S. Kerje, Ö. Carlborg, K. Schütz, L. Jacobsson, C. Hartmann, P. Jensen, and L. Andersson, in prep.). In total, our study indicates that selection for increased growth has acted on a large number of genomic loci. Our studies further indicate that epistasis is important for early growth, that is, during the period where the foundation for rapid growth is set by the development of internal organs, and that epistasis is less important for later growth involving the main deposition of body tissues.

\section{METHODS}

\section{Animals}

A red junglefowl $\times$ White Leghorn population was bred from one red junglefowl male and three White Leghorn females. The $F_{1}$ population was composed of four males and $37 \mathrm{fe}-$ males, and the mapping population consisted of $852 \mathrm{~F}_{2}$ animals. The animals were raised in six separate batches as described by Schütz et al. (2002).

\section{Analysis of Growth Traits}

The weights of the animals were measured at $1,8,46,112$, and 200 days of age. Four estimates of growth rates, 1-8, 8-46, $46-112$, and $112-200$ days of age were calculated as the difference between the body weight recordings at these times.

\section{DNA Isolation and Genetic Marker Analysis}

Blood samples were collected from all $\mathrm{F}_{2}$ individuals, their parents $\left(\mathrm{F}_{1}\right)$, and grandparents $\left(\mathrm{F}_{0}\right)$, and DNA was isolated using the DNeasy 96 Tissue Kit (Qiagen) for mouse tails, with some modifications. All animals were genotyped for 105 ge- 
netic markers evenly distributed in the genome as described in detail elsewhere (S. Kerje, Ö. Carlborg, K. Schütz, L. Jacobsson, C. Hartmann, P. Jensen, and L. Andersson, in prep.). Linkage maps for 25 autosomal linkage groups were computed with the CRI-MAP software (Green et al. 1990). The sex-averaged map spanned $2563 \mathrm{cM}$, and the average marker spacing was $24.4 \mathrm{cM}$. The current search for epistatic QTL did not include analyses of the $\mathrm{Z}$ chromosomes.

\section{QTL Mapping}

Mapping and significance testing for QTL were performed by a slightly modified version of the method for QTL mapping and significance testing described by Carlborg and Andersson (2002). The changes were made to limit the number of randomization tests needed for this study and thus improve the computational efficiency of the strategy. The mapping strategy used included three steps.

\section{Step 1. Forward Selection Genome Scan}

First, QTL were mapped by their marginal genetic effects using the ordinary least-squares-based method for mapping QTL in out-bred line crosses described by Haley et al. (1994). The additive and dominance regression indicator variables for the most significant single QTL in this scan were added as cofactors to the model used for the scan, and a new genome scan was performed using the updated model. This procedure is repeated until no additional significant QTL can be detected. Statistical significance is assessed by randomization testing (Churchill and Doerge 1994) using a 5\% genome-wide threshold for significant and a $20 \%$ genome-wide significance threshold for suggestive QTL.

\section{Step 2. Simultaneous Scan for Epistatic QTL Pairs (SIM)}

We used an exhaustive simultaneous search with a two-locus interaction model to screen for pairs of epistatic QTL. The statistical evaluation of detected QTL pairs was done using two randomization tests described by Carlborg and Andersson (2002). The search and randomization testing procedure will, throughout this report, be referred to as SIM. Two alternate randomization tests were used. The first test was used when one quantitative trait locus is significant by its marginal effects. It is a conditional randomization test, testing if the marginal effects of the second QTL and the interaction parameters significantly improve the fit of the model. The second test was used when none of the QTL were significant by their marginal effects. It is an additional randomization test, which tests if the marginal effects for both QTL, together with their interaction parameters, significantly improve the fit of the model. A 5\% genome-wide threshold was used to declare significant and a $20 \%$ genome-wide significance threshold to declare suggestive QTL pairs.

\section{Step 3. Model Selection for Detected QTL Pairs}

We used a randomization test (Carlborg and Andersson 2002) to select an additive/dominance or an epistatic model for all significant and suggested pairs of QTL in the forward selection and the simultaneous mapping step.

\section{Multiple Regression Modeling}

The indicator regression variables for all loci and interactions detected by the QTL mapping procedures were entered into a multiple regression model to obtain the adjusted sums of squares in order to assess their contribution to the phenotypic variance. The regressions were fit using the SAS software (SAS 1990).

\section{ACKNOWLEDGMENTS}

We thank Lotta Rydmer, Kjell Andersson, Camilla Hartmann, and Måns Tufvesson for valuable discussions regarding chicken genetics; Göran Björnhag and Clas Lilja for discussions regarding avian growth physiology; and Torgny Faxen and Bo Einarsson for helpful discussions and other support. We also thank the National Supercomputing Center (NSC), Linköping, Sweden, for supplying the computer time used for this study. The National Graduate School in Scientific Computing (NGSSC), the Food 21 project (MISTRA), Wallenberg Consortium North, and the AgriFunGen program at the Swedish University of Agricultural Sciences supported the work.

The publication costs of this article were defrayed in part by payment of page charges. This article must therefore be hereby marked "advertisement" in accordance with 18 USC section 1734 solely to indicate this fact.

\section{REFERENCES}

Andersson, L. 2001. Genetic dissection of phenotypic diversity in farm animals. Nat. Rev. Genet. 2: 130-138.

Atchley, W. and Zhu, J. 1997. Developmental quantitative genetics, conditional epigenetic variability and growth in mice. Genetics 147: $765-776$.

Björnhag, G., Knutson, P.-G., and Sperber, I. 1994. Growth-Compendium on the physiology of growth, 3rd ed. (in Swedish). Department of Animal Physiology, Swedish University of Agricultural Sciences, Uppsala, Sweden.

Brockmann, G.A., Kratzsch, J., Haley, C.S., Renne, U., Schwerin, M. and Karle, S. 2000. Single QTL effects, epistasis, and pleiotropy account for two-thirds of the phenotypic F2 variance of growth and obesity in DU6I × DBA/2 mice. Genome Res. 10: 1941-1957.

Carlborg, Ö. and Andersson, L. 2002. The use of randomization testing for detection of multiple epistatic QTL. Genet. Res. 79: $175-184$

Carlborg, Ö., Andersson, L., and Kinghorn, B. 2000. The use of a genetic algorithm for simultaneous mapping of multiple interacting quantitative trait loci. Genetics 155: 2003-2010.

Chase, K., Adler, F.R., and Lark, K.G. 1997. Epistat: A computer program for identifying and testing interactions between pairs of quantitative trait loci. Theor. Appl. Genet. 94: 724-730.

Cheverud, J.M., Rutledge, J., and Atchley, W. 1983. Quantitative genetics of development: Genetic correlations among age-specific trait values and the evolution of ontogeny. Evolution 37: 895-905.

Cheverud, J.M., Routman, E.J., Duarte, F.A., van Swinderen, B., Cothran, K., and Perel, C. 1996. Quantitative trait loci for murine growth. Genetics 142: 1305-1319.

Churchill, G.A. and Doerge, R.W. 1994. Empirical threshold values for quantitative trait mapping. Genetics 138: 963-971.

Falconer, D.S. 1981. Introduction to Quantitative Genetics, 2nd ed., pp. 237. Longman Inc., New York.

Falconer, D.S., Gauld, I., and Roberts, R. 1978. Cell numbers and cell sizes in organs of mice selected for large and small body size. Genet. Res. 31: 387-401.

Fijneman, R.J., De Vries, S.S., Jansen, R.C., and Dermant, P. 1996. Complex interactions of new quantitative trait loci, Sluc1, Sluc2, Sluc3, and Sluc4, that influence the susceptibility to lung cancer in the mouse. Nature Genet. 14: 465-467.

Flint, J. and Mott, R. 2001. Finding the molecular basis of quantitative traits: Successes and pitfalls. Nat. Rev. Genet. 6: $437-445$

Green, P., Falls, K., and Crook, S. 1990. Documentation for CRI-MAP, version 2.4, Washington University School of Medicine, St. Louis, MO.

Haley, C.S., Knott, S.A., and Elsen, J.M. 1994. Mapping quantitative trait loci in crosses between outbred lines using least squares. Genetics 136: 1195-1207.

Hartmann, C. 2002. "Selection for yolk production in laying hens." Ph.D. thesis, Acta Universitatis Agriculturae Sueciae. Agraria 298 Swedish University of Agricultural Sciences.

Jannink, J.-L. and Jansen, R. 2001. Mapping epistatic quantitative trait loci with one-dimensional genome searches. Genetics 157: $445-454$.

Kao, C.-H., Zeng, Z.-B., and Teasdale, R. 1999. Multiple interval mapping for quantitative trait loci. Genetics 152: 1203-1216.

Katanbaf, M.N., Dunnington, E.A., and Siegel, P.B. 1988. Allomorphic relationships from hatching to 56 days in parental lines and F1 crosses of chickens selected 27 generations for high or low body weight. Growth Dev. Aging 52: 11-21. 
Knott, S.A., Marklund, L., Haley, C.S., Andersson, K., Davies, W., Ellegren, H., Fredholm, M., Hansson, I., Hoyheim, B. Lundstrom, K., et al. 1998. Multiple marker mapping of quantitative trait loci in a cross between outbred wild boar and large white pigs. Genetics 149: 1069-1080.

Korstanje, R. and Paigen, B. 2002. From QTL to gene: The harvest begins. Nature Genet. 31: 235-236.

Leamy, L. and Cheverud, J. 1984. Quantitative genetics and the evolution of ontogeny. II. Genetic and environmental correlations among age-specific characters in randombred mice. Growth 48: 339-353.

Lilja, C. 1983. A comparative study of postnatal growth and organ development in some species of birds. Growth 47: 317-339.

Lilja, C. and Marks, H.L. 1991. Changes in organ growth pattern associated with long-term selection for high growth rate in quail. Growth Dev. Aging 55: 219-224.

Lilja, C., Sperber, I., and Marks, H.L. 1985. Postnatal growth and organ development in Japanese quail selected for high growth rate. Growth 49: 51-69.

Mackay, T.F. 2001. Quantitative trait loci in Drosophila. Nat. Rev. Genet. 1: 11-20.

Nir, I., Nitzan, Z., and Mahagna, M. 1993. Comparative growth and development of the digestive organs and of some enzymes in broiler and egg type chicks after hatching. Br. Poult. Sci. 34: $523-532$.
Nitzan, Z., Ben-Avraham, G., Zoref, Z., and Nir, I. 1991. Growth and development of the digestive organs and some enzymes in broiler chicks after hatching. Br. Poult. Sci. 32: 515-523.

Riska, B., Atchley, W., and Rutledge, J. 1984. A genetic analysis of targeted growth in mice. Genetics 107: 79-101.

SAS 1990. SAS user's guide: Statistics. SAS Institute, Cary, NC.

Schütz, K., Kerje, S., Carlborg, Ö., Jacobsson, L., Andersson, L., and Jensen, P. 2002. Analysis of a red junglefowl $\times$ White Leghorn intercross reveals trade-off in resource allocation between behaviour and production traits. Behav. Genet. 32: 423-433.

Shimomura, K., Low-Zeddues, S.S., King, D.P., Steeves, T.D.L., Whiteley, A., Kushla, J., Zemenides, P.D., Lin, A., Vitaterna, M.H., Churchill, G.A., et al. 2001. Genome-wide epistatic interaction analysis reveals complex genetic determinants of circadian behavior in mice. Genome Res. 11: 959-980.

Vaughn, T.T., Pletscher, L.S., Peripato, A., King-Ellison, K., Adams, E., Erikson, C., and Cheverud, J.M. 1999. Mapping quantitative trait loci for murine growth: A closer look at genetic architecture. Genet. Res. 74: 313-322.

Zeng, Z.-B., Liu, J., Stam, L.F., Kao, Z.-H., Mercer, J.M., and Laurie, C.C. 2000. Genetic architecture of a morphological shape difference between two Drosophila species. Genetics 154: 299-310.

Received June 14, 2002; accepted in revised form December 10, 2002. 


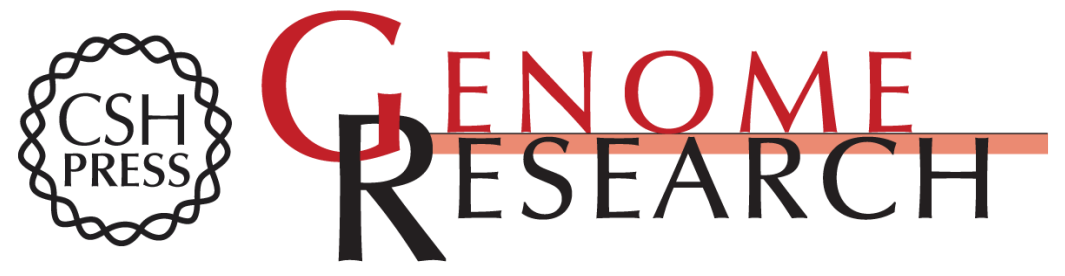

\section{A Global Search Reveals Epistatic Interaction Between QTL for Early Growth in the Chicken}

Örjan Carlborg, Susanne Kerje, Karin Schütz, et al.

Genome Res. 2003 13: 413-421

Access the most recent version at doi:10.1101/gr.528003

Supplemental Material

References

License

Email Alerting Service
http://genome.cshlp.org/content/suppl/2003/03/21/13.3.413.DC1

This article cites 29 articles, 12 of which can be accessed free at: http://genome.cshlp.org/content/13/3/413.full.html\#ref-list-1

Receive free email alerts when new articles cite this article - sign up in the box at the top right corner of the article or click here.

\section{Affordable, Accurate Sequencing.}

